\title{
Conventional and Multivariate Statistical Methods for Evaluation of In vitro Dissolution Similarity of Bisoprolol Film-coated Tablets
}

\author{
Ivana Mitrevska ${ }^{1,}$,, Ljupco Pejov ${ }^{2,3}$, Marija Jovanovska ${ }^{1}$, Suzan Memed-Sejfulah ${ }^{1}$, \\ Katerina Brezovska ${ }^{4}$, Aneta Dimitrovska ${ }^{4}$, Sonja Ugarkovic ${ }^{1}$ \\ ${ }^{1}$ Research \& Development, Alkaloid AD, Skopje, R. North Macedonia \\ ${ }^{2}$ Department of Chemistry, Bioscience and Environmental Engineering, Faculty of Science and Technology, University of Stavanger, Stavanger, \\ Norway \\ ${ }^{3}$ Institute of Chemistry, 'Ss. Cyril and Methodius" University, Faculty of Natural Sciences and Mathematics, Skopje, R. North Macedonia \\ ${ }^{4}$ Institute of Applied Chemistry and Pharmaceutical Analysis, 'Ss. Cyril and Methodius” University, Faculty of Pharmacy, Skopje, R. North \\ Macedonia
}

Email address:

imickoska@alkaloid.com.mk (I. Mitrevska)

${ }^{*}$ Corresponding author

\section{To cite this article:}

Ivana Mitrevska, Ljupco Pejov, Marija Jovanovska, Suzan Memed-Sejfulah, Katerina Brezovska, Aneta Dimitrovska, Sonja Ugarkovic. Conventional and Multivariate Statistical Methods for Evaluation of In vitro Dissolution Similarity of Bisoprolol Film-coated Tablets. International Journal of Pharmacy and Chemistry. Vol. 6, No. 2, 2020, pp. 16-25. doi: 10.11648/j.ijpc.20200602.12

Received: June 9, 2020; Accepted: June 20, 2020; Published: July 4, 2020

\begin{abstract}
In vitro dissolution testing is an important tool used for pharmaceutical development and approval of generic medicinal product, playing a pivotal role in regulatory decision-making. This study includes evaluation and comparative analysis of in vitro dissolution profiles of Bisoprolol film-coated tablets and in vitro dissolution profile of a reference medicinal product, using several model-independent and model-dependent statistical methods. The evaluated medicinal product belongs to BCS Class I (high solubility, high permeability). The similarity testing of dissolution profile is performed on the highest strength of the dosage form, in accordance with the regulatory requirements for bioequivalence study. Obtained results have shown that in vitro comparative dissolution analysis using pair-wise independent-model procedures, such as difference $\left(f_{l}\right)$ and similarity $\left(f_{2}\right)$ factors are not suitable, because one of the requirements (not more than one mean value dissolves more than $85 \%$, for any of the formulations) was not fulfilled. Therefore, the comparison of the similarity of dissolution profiles was performed using Ratio test methodology and multivariate model-independent approach based on generalized statistical distance (Mahalanobis distance). Furthermore, other model-dependent approaches coupled to multivariate statistics (Weibull) were applied. The obtained results from the performed analysis indicated a significant similarity of the compared in vitro dissolution profiles between the tested batches from bisoprolol film-coated tablets and reference medicinal product). Implemented statistical methods can be considered as a regulatory accepted concept for evaluation of in vitro similarity of generic medicines.
\end{abstract}

Keywords: Generic Medicinal Product, In vitro Dissolution Similarity, Model-dependent Methods, Model-independent Methods, Bisoprolol Film-coated Tablets

\section{Introduction}

In vitro dissolution testing plays a critical role in the life cycle of a generic medicinal product. The dissolution method should be sufficiently robust and reproducible for daily operations, capable of being transferred between laboratories, and adequately discriminating to distinguish any changes that could affect in vivo performance of the product [1].

Dissolution studies are usually associated with the bioavailability of the active pharmaceutical ingredient (API) in the body. The relevance of in vitro dissolution studies of medicinal products has been increasing over time, and these studies even replace in vivo studies in certain circumstances 
[2].

According to the scientific principles of the Biopharmaceutical Classification System (BCS) and regulatory framework, rapid dissolved oral solid dosage forms containing BCS class 1 and 3 drugs can provide regulatory relief on the biowaivers basis. An IR drug product is considered rapidly dissolving when no less than $85 \%$ of the labeled amount of the drug substance dissolves within 30 minutes [3].

In addition, the World Health Organization (WHO) recommended biowaiving of in vivo bioequivalence (BE) studies for some poorly soluble but highly permeable weakly acidic compounds capable of meeting the "rapid dissolution" criteria at $\mathrm{pH} 6.8$ and showing similar dissolution profile with reference product at $\mathrm{pH} 1.2,4.5$, and 6.8. In view of the fact that this concept was not widely accepted by other regulatory bodies due to a lack of scientific, credible and convincing evidence, WHO has not provided further support in developing the latest $\mathrm{BE}$ strategies [4].

Establishing in vivo-in vitro (IVIVC) correlations can help reduce development costs, accelerate product development and reduce the need for bioavailability / bioequivalence studies on human volunteers. Moreover, for certain drugs with BCS class 2 drugs where absorption is limited by dissolution rate, BCS-based biowaiver does not appear to be straightforward.

In line with regulatory agencies, the most commonly used and easiest approach to comparing dissolution profiles is by determining the similarity factor $f_{2}$ developed by Moore and Flanner which by its nature has no strong statistical basis. [5-7].

Similarity of the dissolution is assessed by in vitro comparison of the dissolution profiles (usually percent of dissolved active substance $v s$. time) and it has been discussed in the EMA and FDA guidelines [5-7]. Significant comparability is achieved when the dissolution profiles are adequately characterized in terms of the number of time points and permitted data variability. Dissolution similarity for regulatory purposes should be assessed in at least three media, preferably: $\mathrm{pH} 1.2, \mathrm{pH} 4.5$ and $\mathrm{pH} 6.8$ (additionally specification media can be included if it is different). For IR formulations, comparison at 15 minutes becomes essential and should be included in the profiles as it is related to the physiological gastric emptying time.

Since the 90s, regulatory agencies and scientific researchers have increasingly suggested the use of new mathematicalstatistical methods to prove in vitro dissolution similarity [8]. Hence, in cases when the $f_{2}$ statistics is not applicable, the guidelines address the possibility for using alternative statistical methodologies for dissolution profiles comparison, using model-dependent or model-independent methods [9, $10]$.

This concept has been further elaborated in few studies, but not many publications demonstrate real case studies on using alternative statistical methodologies for comparative dissolution analysis. Therefore, it would be highly beneficial to further elaborate this concept and present a detailed regulatory approved approach. This study includes evaluation and comparative analysis of in vitro dissolution profiles of Bisoprolol film-coated tablets and in vitro dissolution profile of a reference medicinal product, using several model-independent and model-dependent statistical methods. The evaluated medicinal product belongs to BCS Class I (high solubility, high permeability).

\section{Materials and Methods}

The dissolved amount is determined with HPLC method. Reference medicinal products (Concor $10 \mathrm{mg}$ film-coated tablets) were purchased at EU market and test products (Bisoprolol $10 \mathrm{mg}$ film-coated tablets) were manufactured by Alkaloid AD Skopje.

\subsection{Determination of Saturation Solubility}

The saturation solubility of bisprolol fumarate was determined in the following media: buffer $\mathrm{pH} 1.2(\mathrm{NaCl}, \mathrm{HCl})$, pH 4.5 acetate buffer and pH 6.8 phosphate. Experiments were conducted in triplicate using a shake-flask protocol with sample quantification by UV-spectroscopy. Excess amount of bisoprolol fumarate was added in each medium in a $250 \mathrm{~mL}$ shake-flask producing a suspension that was subsequently agitated for 12 hours on an electronic shake-plate. After agitation, the sample was left for a sedimentation period of 12 hours before an aliquot of the supernatant solution was extracted by pipette, filtered under vacuum through a $0.2 \mu \mathrm{m}$ PVDF filter plate. The solubility was subsequently determined and sample was analyzed by UV-spectroscopy over a wavelength range of $250 \mathrm{~nm}-270 \mathrm{~nm}$.

\subsection{Dissolution Studies}

Testing is carried out at $37 \pm 0.5^{\circ} \mathrm{C}$ and $75 \mathrm{r} / \mathrm{min}$ using calibrated Apparatus II (paddle) with $900 \mathrm{~mL} \pm 1 \%$ of each medium. Sampling for all dissolution tests is performed at 10, 15,20 and 30 minutes. In all cases, media were deaerated and filtered through $0.45 \mu \mathrm{m}$ regenerated cellulose membrane filter. The samples were taken manually through a $10 \mathrm{~mL}$ syringe, connected to a stainless steel sampling cannula. At each sampling time, $10 \mathrm{~mL}$ of medium is removed and filtered through a $0.20 \mu \mathrm{m}$ regenerated cellulose membrane filter. Trials are performed with twelve (12) film-coated tablets and the obtained values are used for data analysis. A Bisoprolol tablet is officinal pharmacopoeia product (USP 31/NF 26) where buffer $\mathrm{pH} 1.2(\mathrm{HCl}, \mathrm{NaCl})$ is listed as a dissolution medium of choice for combined medicinal product with Hydrochlorothiazide [11]. Additionally, comparative dissolution testing in $\mathrm{pH} 4.5$ acetate buffer and $\mathrm{pH} 6.8$ phosphate buffer were performed. Dissolution media: $\mathrm{pH} 1.2$ $(\mathrm{HCl}, \mathrm{NaCl})$, acetate buffer $\mathrm{pH} 4.5$ and phosphate buffer $\mathrm{pH}$ 6.8 were prepared according to directions in European Pharmacopeia monograph (Ph. Eur., 5.17., 2010).

\subsection{Quantitative Analysis}

Samples obtained from dissolution experiments were 
quantitatively analyzed using a HPLC method previously validated. The column Spherisorb Pheny 1, $125 \mathrm{~mm} \times 4.0 \mathrm{~mm}$, $5 \mu \mathrm{m}$, is maintained at ambient temperature, with UV detector wavelength of $227 \mathrm{~nm}$. The isocratic mode involves two mobile phases buffer pH 3.0 (2.0 mL Triethylamine in 1000 $\mathrm{mL}$ water) as solvent $\mathrm{A}$ and acetonitrile as solvent $\mathrm{B}$ in ratio, $80 / 20(\mathrm{~V} / \mathrm{V} \%)$. The flow rate is set to $1.2 \mathrm{~mL} / \mathrm{min}$. The analysis run time is 5 minutes and the injection volume is 20 $\mu \mathrm{L}[11]$.

\subsection{Applied Statistical Methodology to Compare Dissolution Profiles}

A preliminary analysis of dissolution data was carried out by plotting of "mean dissolution profiles" (test vs. approved, i.e. reference) with error bars extending to $\pm \sigma$, as well as with 95\% confidence intervals. An overlap between 95\% confidence intervals evidenced at each time point was considered as a strong indication of the similarity between the investigated time series (though, of course, not definite or quantitative).

We have proceeded our analysis with two types of approaches which have been proposed in the literature for this purpose: model-independent and model-dependent ones.

\subsubsection{Univariate Model-independent Approaches}

Difference $\left(f_{1}\right)$ and similarity $\left(f_{2}\right)$ factors are computed by the standard formulas [1-3]:

$$
\begin{gathered}
f_{1}=100 \cdot\left[\frac{\sum_{t=1}^{n}\left|F_{R, t}-F_{T, t}\right|}{\sum_{t=1}^{n} F_{R, t}}\right], \\
f_{2}=50 \cdot \log \left\{100 \cdot\left[1+\frac{1}{n} \sum_{t=1}^{n}\left(F_{R, t}-F_{T, t}\right)^{2}\right]^{-0.5}\right\},
\end{gathered}
$$

In (1) and (2), $F_{R, t}$ and $F_{T, t}$ denote the percentages of dissolved reference $(\mathrm{R})$ and test $(\mathrm{T})$ samples, respectively.

These two quantities were computed considering both the "mean dissolution profiles" of the test and reference samplesi.e. as "mean test" vs. "mean reference" $[12,13]$.

In the latter case, also the standard error was computed to get a better insight into the data variability. However, note that in the case of presently analyzed data sets, an essential prerequisite for the similarity factor $f 2$ to be used as a valid indicator of similarity between the compared sets is not fulfilled. Namely, in the case of test products, more than one mean value of $\mathrm{F}$ is larger than $85 \%$. The computed $f 2$ values are, therefore, in this context presented just for comparison purposes, and not with an aim to derive conclusions concerning similarity of dissolution profiles.

Of the other model-independent methodologies, the ratio test approaches is established. The ratio tests of the percent of dissolved test/ratio samples (PD), area under the concentration curve (AUC) as well as the average dissolution time (ADT) are implemented. These ratios were calculated (3) between the test and ratio average values at each time point; for example, in the case of PD [14]:

$$
R(\mathrm{PD})_{T / R, t}=\frac{\left\langle F_{T}\right\rangle_{t}}{\left\langle F_{R}\right\rangle_{t}},
$$

Further, the standard errors (SE) were estimated by the formula:

$$
S E_{R(\mathrm{PD})}=\frac{\left\langle F_{T}\right\rangle_{t}}{\left\langle F_{R}\right\rangle_{t}} \cdot \sqrt{\left(\frac{S E_{T, t}}{\left\langle F_{T}\right\rangle_{t}}\right)^{2}+\left(\frac{S E_{R, t}}{\left\langle F_{R}\right\rangle_{t}}\right)^{2}},
$$

$\mathrm{SE}_{\mathrm{T}, \mathrm{t}}$ and $\mathrm{SE}_{\mathrm{R}, \mathrm{t}}$ being the standard errors of the test and reference samples at time point $t$, respectively) and the $90 \%$ confidence intervals (CIs) were calculated as:

$$
C I=\frac{\left\langle F_{T}\right\rangle_{t}}{\left\langle F_{R}\right\rangle_{t}} \pm t_{\left(0.1, n_{R}+n_{T}-2\right)} \cdot S E_{R(P D)},
$$

In the previous equation (5), $n_{T}$ and $n_{R}$ denote the number of data in the cases of test and reference samples correspondingly, while $t_{\left(0.1, n_{R}+n_{T}-2\right)}$ is the value of $t$-variable with $n_{R}+n_{T}-2$ degrees of freedom and a confidence limit of $90 \%$. Analogous procedures were adopted in the case of AUC and ADT ratios. Note that these three procedures are particularly useful in cases when most of the drug has been dissolved in a relatively short time.

\subsubsection{Multivariate Model-independent Approach Based on Generalized Statistical Distance}

The Mahalanobis distance DM is generalized as a measure of multivariate statistical methods. Each of the twelve measurements at four time points in the case of test and reference medicinal products thus constitutes a particular realization of a stochastic $1 \times 4$ vector $x$ in $\mathfrak{R}^{4}$. Further, $\mathrm{p} \times \mathrm{n}$ $(12 \times 4$ in our case) matrix $M$ containing each particular realization of the vector $\mathrm{x}$ is defined. The elements of matrix $\mathrm{M}$ are denoted as $x_{i, j}$, where $\mathrm{i}=1, \ldots 12$, while $j=1, \ldots .4$. The vector $x$ is denoted and the data matrix $M$ composed by measurements of the reference and test samples by $x_{\mathrm{R}(\mathrm{T})}$ and $\mathrm{M}_{\mathrm{R}(\mathrm{T})}$ respectively [15].

After collecting the matrices $\mathrm{M}_{\mathrm{R}(\mathrm{T})}$ and vectors $x_{\mathrm{R}(\mathrm{T})}$, the variance-covariance matrices of the reference and test samples are computed, as well as the mean (average)-vectors $x_{\mathrm{av}, \mathrm{R}(\mathrm{T})}$. The later quantities are defined with the following elements:

$$
x_{j, \text { av. }}=\frac{1}{p} \sum_{i=1}^{p} x_{i j}
$$

i.e.:

$$
\mathrm{x}_{\mathrm{ay.}, \mathrm{R}(\mathrm{T})}=\mathrm{E}\left[\left(\mathrm{x}_{\mathrm{R}(\mathrm{T})}\right)\right],
$$

where $\mathrm{E}$ denotes the mathematical expectation. 
The variance-covariance matrices $\Sigma_{\mathrm{R}(\mathrm{T})}$ are, on the other hand, defined with [14]:

$$
\sum_{\mathrm{R}(\mathrm{T})}=\mathrm{E}\left[\left(\mathrm{x}_{\mathrm{R}(\mathrm{T})}-\mathrm{x}_{\mathrm{ay}, \mathrm{R}(\mathrm{T})}\right)\left(\mathrm{x}_{\mathrm{R}(\mathrm{T})}-\mathrm{x}_{\mathrm{ay}, \mathrm{R}(\mathrm{T})}\right)^{\mathrm{T}}\right],
$$

where $\mathrm{T}$ denotes transposition of the difference vectors (or "centered" x-vectors). Subsequently, the "pooled" (across the reference and test samples) variance-covariance matrix is computed and defined as:

$$
\sum_{\text {pooled }}=0.5\left(\sum_{\mathrm{R}}+\sum_{\mathrm{T}}\right)
$$

Mahalanobis distance $\mathrm{D}_{\mathrm{M}}$ is computed as:

$$
D_{\mathrm{M}}=\sqrt{\left(\mathrm{x}_{\mathrm{T}}-\mathrm{x}_{\mathrm{R}}\right)^{\mathrm{T}} \Sigma_{\text {pooled }}^{-1}\left(\mathrm{x}_{\mathrm{T}}-\mathrm{x}_{\mathrm{R}}\right)},
$$

where $\Sigma_{\text {pooled }}^{-1}$ is the inverse of the pooled variance-covariance matrix. To define the limiting $D_{M}$ value $-D_{M \text {,lim. ("the }}$ similarity limit", in the usual parlance), $1 \times 4$ vector $\mathrm{x}_{\mathrm{lim}}$. is defined, the elements of which are the predefined limits expressed as maxima of the tolerable average distances at all time points.

$$
D_{\mathrm{M}, \mathrm{lim} .}=\sqrt{\mathrm{x}_{\text {lim. }}^{\mathrm{T}} \Sigma_{\text {pooled }}^{-1} \mathrm{x}_{\text {lim. }}},
$$

Assuming that the data follow multivariate normal distribution, we have subsequently calculated the $90 \%$ confidence region for the "true" difference between the population-based mean vectors $\mu_{\mathrm{T}}-\mu_{\mathrm{R}}$ from the condition:

$$
K \cdot\left[\mu-\left(\mathrm{x}_{\mathrm{T}}-\mathrm{x}_{\mathrm{R}}\right)\right]^{\mathrm{T}} \Sigma_{\text {pooled }}^{-1}\left[\mu-\left(\mathrm{x}_{\mathrm{T}}-\mathrm{x}_{\mathrm{R}}\right)\right] \leq F_{\left(d, p_{\mathrm{T}}+p_{\mathrm{R}}-d-1,0.9\right)},
$$

In the previous equation (12), $K$ is a scaling factor, defined with:

$$
K=\frac{p_{\mathrm{T}} \cdot p_{\mathrm{R}}}{p_{\mathrm{T}}+p_{\mathrm{R}}} \cdot \frac{p_{\mathrm{T}}+p_{\mathrm{R}}-d-1}{\left(p_{\mathrm{T}}+p_{\mathrm{R}}-2\right) \cdot d},
$$

where $p_{T}$ and $p_{R}$ are the number of measurements (realizations of the vector components) in the case of test and reference samples respectively (both of which are 12 in the present case), while $d$ is the dimension of the vectors $x_{R(T)}(d=4$ in the present case). $F_{(\mathrm{d}, \mathrm{pT}+\mathrm{pR}-\mathrm{d}-1,0.9)}$, on the other hand, is the 90 percentile of the F-distribution with $d$ and $p_{T}+p_{R}-d-1$ degrees of freedom. Multiplication by the scaling factor $\mathrm{K}$ in (12) is in fact required in order to obtain a new statistics which is directly comparable to a standard F-distribution. The confidence region calculated is denoted from the condition (12) by:

$$
\left(D_{\mathrm{M}}^{\mathrm{L}}, D_{\mathrm{M}}^{\mathrm{H}}\right)
$$

where "L" and "H" imply the "lower" and "higher" limit of the interval. The conclusion about the similarity of the vectors $\mathrm{x}_{\mathrm{R}}$ and $\mathrm{x}_{\mathrm{T}}$ is derived, if the condition:

$$
D_{\mathrm{M}}^{\mathrm{H}} \leq D_{\mathrm{M}, \mathrm{lim} .},
$$

is fulfilled, i.e. if the higher limit of the interval defined with (14) is less then or equal to the limiting value of the Mahalanobis distance.

\subsubsection{Multivariate Model-dependent Approach}

For the purpose of the present study the model-dependent approach is adopted. This procedure covered the most widely used functions which are both kinetically and mechanistically based. The goodness-of-fit is judged by the $R^{2}$ values, adjusted $R^{2}$ and Akaike information criterion (AIC). The best fit has been obtained by the two-parameter Weibull model function of the form:

$$
F(t)=100 \cdot\left[1-\exp \left(-\frac{t^{\beta}}{\alpha}\right)\right],
$$

Subsequently, the model parameters $\alpha$ and $\beta$ have been obtained for all available series of data (for both test and reference samples) by non-linear least-squares fitting procedure [16]. Furthermore, a multivariate similarity test of the model parameters between reference and test samples is performed.

Statistical analysis are carried out in parallel (due to cross-checking of the results) with the Microcal Origin Pro v. 9 [17], Wolfram Mathematica 10 [18], as well as Microsoft Excel 2007 [19].

\section{Results and Discussion}

To determine the sink conditions, knowledge of the solubility profile of $\mathrm{AC}$ at various $\mathrm{pH}$ is required. Regarding to this, the requirement for sink conditions should be satisfied in a $\mathrm{pH}$ medium where in vivo dissolution and / or absorption of the active substance is expected or, in other words, in the specification medium [20]. Bisoprolol tablet is officinal pharmacopoeia product (USP31/NF 26) where buffer $\mathrm{pH} 1.2$ $(\mathrm{HCl}, \mathrm{NaCl})$ is listed as a dissolution medium of choice for combined medicinal product with Hydrochlorothiazide. Therefore, to define the solubility of bisoprolol fumarate, a shake-flask protocol with sample quantification by UV-spectroscopy was carried out. To determine the solubility, $3.0 \mathrm{~mL}$ of each tested buffers was added to $7.2 \mathrm{~g}$ of pure bisoprolol fumarate, producing a suspension that was subsequently agitated for 12 hours on an electronic shake-plate. After agitation, the sample was left for a sedimentation period of 12 hours before an aliquot of the supernatant solution was extracted by pipette, filtered under vacuum through a $0.2 \mu \mathrm{m}$ PVDF filter plate.

Bisoprolol fumarate exhibited higher solubility in all tested media. The solubility in the recommended media $\mathrm{pH} 1.2$ was subsequently determined as $1674 \mathrm{mg} / \mathrm{mL}$ from the measured absorption of the supernatant solution (Figure 1) which is more above from the theoretically calculated sink concentration $(3 \times 10 \quad \mathrm{mg} / 250 \quad \mathrm{~mL}=0.12 \quad \mathrm{mg} / \mathrm{mL})$. Consequently, it was concluded that the sink conditions were 
satisfied for the recommended $\mathrm{pH} 1.2$ dissolution medium. Two general approaches for comparison of in vitro dissolution profiles of four industrial batches of Bisoprolol film-coated tablets and reference (Concor film-coated tablets) medicinal product are used: model-dependent and model-independent. The results from experimental dissolution studies expressed as a percentage of released API vs. time and descriptive analyses for reference and test medicinal products of bisoprolol are given in Table 1. The in vitro dissolution profiles of the tablets are shown in Figure 2. Each data point represents a mean of twelve measurements for each sample. In this work, dissolution profiles are compared by different methods belonging to one of three main classes: univariate model-independent, multivariate model-independent and multivariate model-dependent methods.

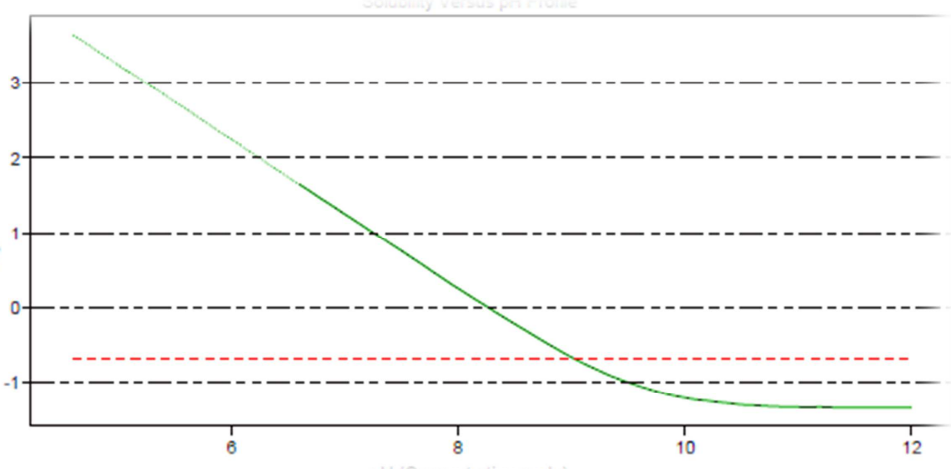

Figure 1. Solubility vs. pH profile of bisoprolol fumarate.

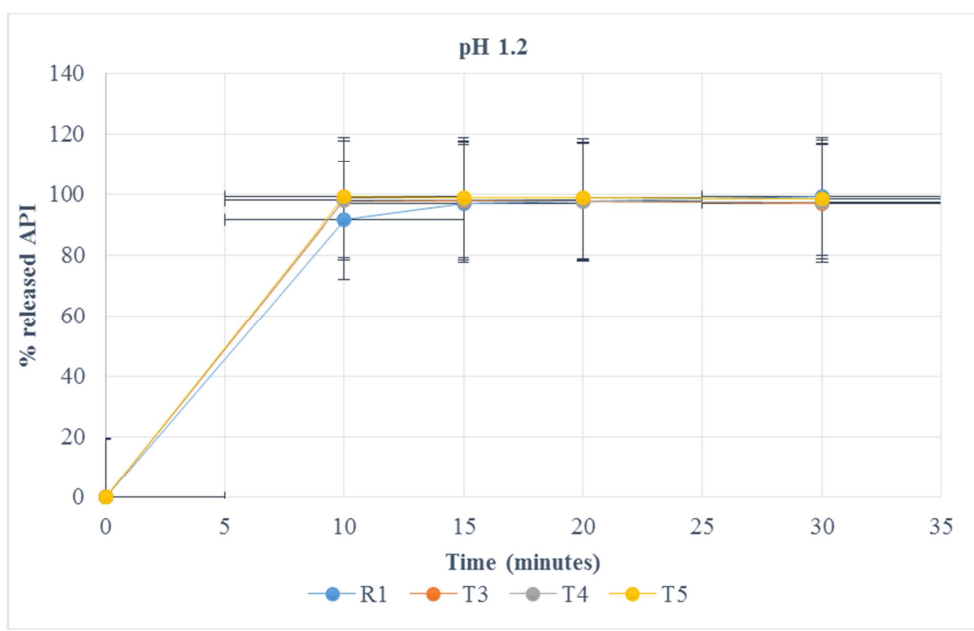

(a) In vitro dissolution profiles in medium $p H 1.2$.

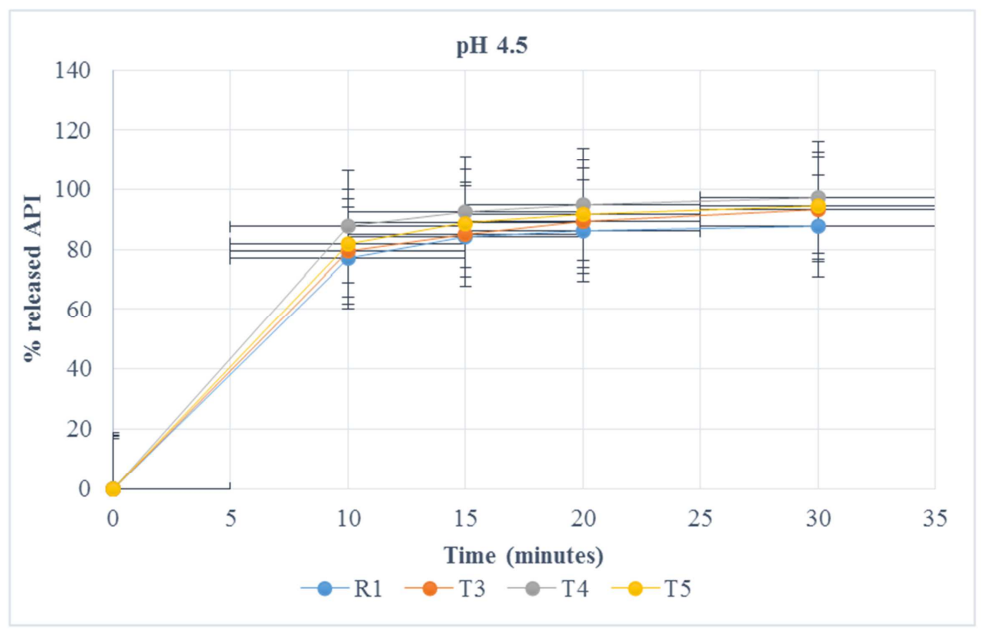

(b) In vitro dissolution profiles in medium $\mathrm{pH} 4.5$. 


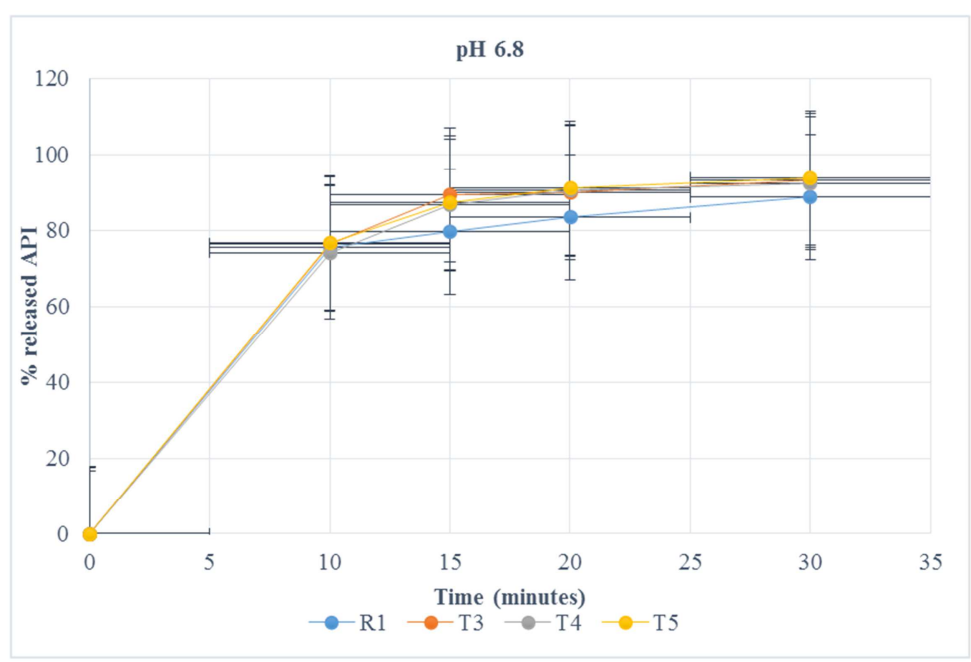

(c) In vitro dissolution profiles in medium $\mathrm{pH} 6.8$.

Figure 2. In vitro dissolution profiles of reference $(R)$ and test $(T)$ medicinal products.

\subsection{Univariate Model-independent Approaches}

The $f_{l}$ (difference factor) is proportional to the average difference between the two profiles, whereas $f_{2}$ (similarity factor) is inversely proportional to the average squared difference between the two profiles. Generally, $f_{1}$ values up to $15(0-15)$ and $f_{2}$ values greater than $50(50-100)$ ensure similarity of the two curves. The values of $f_{1}$ and $f_{2}$ factors for test $v s$. reference samples are calculated from the means of percent dissolved at each time point (Table 1) by using (1) and (2) and listed in Table 2 for all dissolution media.

Calculated $f_{1}$ and $f_{2}$ values (Table 2) from dissolution profiles of test $v s$. reference samples ensured pharmaceutical equivalence. Nevertheless, the $f_{2}$ values in this context are presented only for comparison purposes, and are not applied for making definitive conclusions concerning similarity of dissolution profiles.

The results obtained from the first time point (10 minutes) of the dissolution medium buffer $\mathrm{pH} 1.2$ are already relatively high and the product dissolved more than $85 \%$ for 15 minutes. For that reason, further mathematical calculations are not needed and products can be considered equivalent to each other. At $\mathrm{pH} 4.5$ and $\mathrm{pH} 6.8$, for all generated profiles, more than one mean value above $85 \%$ for any of the tested batches is observed, creating a consequence that the $f_{2}$ statistics for determining profile similarity is not applicable. In accordance to the guideline, in such cases alternative statistical methodologies can be employed for demonstrating dissolution similarity.

Table 1. In vitro dissolution profiles of reference $(R)$ and test $(T)$ medicinal products.

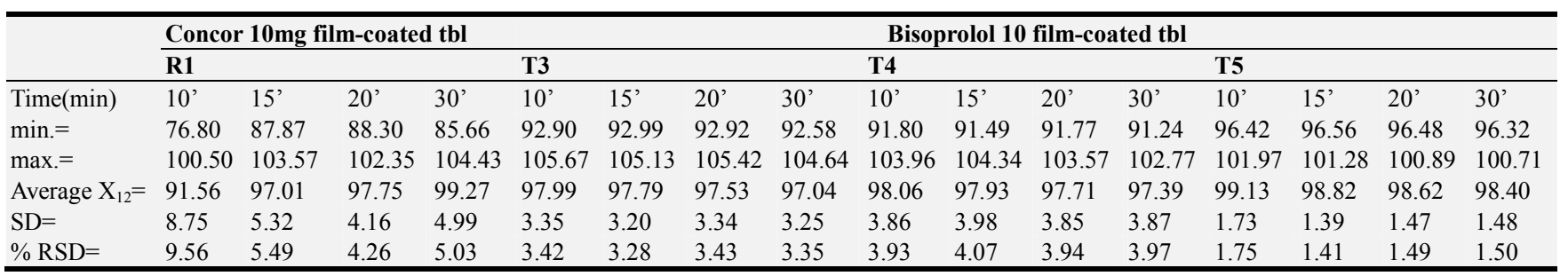

a. In vitro dissolution profiles of reference $(\mathrm{R})$ and test $(\mathrm{T})$ medicinal products in buffer $\mathrm{pH} 1.2(\mathrm{NaCl}, \mathrm{HCl})$.

\begin{tabular}{|c|c|c|c|c|c|c|c|c|c|c|c|c|c|c|c|c|}
\hline & \multicolumn{8}{|c|}{ Concor $10 \mathrm{mg}$ film-coated tbl } & \multicolumn{8}{|c|}{ Bisoprolol 10 film-coated tbl } \\
\hline & R1 & & & & T3 & & & & T4 & & & & T5 & & & \\
\hline Time(min) & $10^{\prime}$ & $15^{\prime}$ & 20 ' & 30 ' & 10 ' & $15^{\prime}$ & 20 ' & 30 ' & 10 ' & $15^{\prime}$ & 20 ' & 30 ' & 10 ' & 15 ' & $20^{\prime}$ & 30 ' \\
\hline $\min .=$ & 58.41 & 76.03 & 76.33 & 68.83 & 61.65 & 71.40 & 78.26 & 85.44 & 71.60 & 84.89 & 88.79 & 92.79 & 59.49 & 68.77 & 75.07 & 83.84 \\
\hline $\max .=$ & 92.58 & 94.08 & 93.89 & 96.42 & 99.37 & 99.68 & 100.12 & 100.88 & 105.18 & 106.05 & 107.27 & 108.12 & 93.62 & 95.36 & 98.93 & 100.0 \\
\hline Average $\mathrm{X}_{12}=$ & 77.11 & 84.44 & 86.29 & 87.74 & 79.47 & 85.04 & 89.46 & 93.44 & 87.65 & 92.50 & 95.00 & 97.41 & 81.99 & 88.80 & 91.78 & 94.69 \\
\hline $\mathrm{SD}=$ & 8.75 & 5.32 & 4.16 & 4.99 & 10.66 & 8.26 & 6.93 & 5.16 & 9.81 & 6.49 & 5.32 & 4.12 & 10.26 & 7.65 & 6.22 & 4.12 \\
\hline$\% \mathrm{RSD}=$ & 9.56 & 5.49 & 4.26 & 5.03 & 13.42 & 9.72 & 7.74 & 5.53 & 11.19 & 7.01 & 5.60 & 4.23 & 12.51 & 8.62 & 6.78 & 4.36 \\
\hline
\end{tabular}

b. In vitro dissolution profiles of reference $(\mathrm{R})$ and test $(\mathrm{T})$ medicinal products in phosphate buffer $\mathrm{pH} 4.5$.

\begin{tabular}{|c|c|c|c|c|c|c|c|c|c|c|c|c|c|c|c|c|}
\hline & \multicolumn{4}{|c|}{ Concor 10mg film-coated tbl } & \multicolumn{12}{|c|}{ Bisoprolol 10 film-coated tbl } \\
\hline & R1 & & & & T3 & & & & T4 & & & & T5 & & & \\
\hline Time(min) & $10^{\prime}$ & $15^{\prime}$ & $20^{\prime}$ & $30^{\prime}$ & $10^{\prime}$ & $15^{\prime}$ & $20^{\prime}$ & $30^{\prime}$ & $10^{\prime}$ & $15^{\prime}$ & $20^{\prime}$ & $30^{\prime}$ & $10^{\prime}$ & $15^{\prime}$ & $20^{\prime}$ & $30^{\prime}$ \\
\hline $\min .=$ & 68.32 & 72.54 & 77.67 & 83.62 & 68.75 & 78.20 & 83.14 & 86.36 & 49.61 & 74.86 & 79.34 & 84.70 & 50.72 & 76.76 & 81.20 & 86.85 \\
\hline $\max .=$ & 85.91 & 92.01 & 92.49 & 94.15 & 85.61 & 97.48 & 98.53 & 98.96 & 87.07 & 97.06 & 97.98 & 98.58 & 88.98 & 97.43 & 99.02 & 100.48 \\
\hline
\end{tabular}




\begin{tabular}{|c|c|c|c|c|c|c|c|c|c|c|c|c|c|c|c|c|}
\hline \multirow[b]{3}{*}{$\begin{array}{l}\text { Average } \\
\mathrm{X}_{12}=\end{array}$} & \multicolumn{8}{|c|}{ Concor 10mg film-coated tbl } & \multicolumn{8}{|c|}{ Bisoprolol 10 film-coated tbl } \\
\hline & R1 & & & & T3 & & & & T4 & & & & T5 & & & \\
\hline & 75.39 & 79.63 & 83.32 & 88.66 & 76.48 & 89.32 & 89.98 & 93.05 & 74.16 & 86.65 & 90.53 & 92.33 & 76.57 & 87.37 & 91.03 & 93.85 \\
\hline $\mathrm{SD}=$ & 4.67 & 5.66 & 6.15 & 5.23 & 4.23 & 6.29 & 5.16 & 4.76 & 9.85 & 7.42 & 5.53 & 4.58 & 10.96 & 7.21 & 5.21 & 4.23 \\
\hline$\% \mathrm{RSD}=$ & 4.98 & 7.50 & 7.72 & 6.28 & 5.53 & 7.04 & 5.73 & 5.12 & 13.28 & 8.56 & 6.11 & 4.96 & 14.31 & 8.25 & 5.72 & 5.53 \\
\hline
\end{tabular}

c. In vitro dissolution profiles of reference $(\mathrm{R})$ and test $(\mathrm{T})$ medicinal products in phosphate buffer $\mathrm{pH} 6.8$.

Table 2. The values of $f_{1}$ and $f_{2}$ parameters computed from the experimental dissolution data for reference $(R)$ and test $(T)$ medicinal products.

\begin{tabular}{|c|c|c|c|}
\hline Medium 4.5 & T3 & T4 & T5 \\
\hline$\overline{f_{1}}$ & 3.53 & 11.02 & 6.46 \\
\hline$f_{2}$ & 72.06 & 51.46 & 62.60 \\
\hline \multicolumn{4}{|l|}{ Medium 6.8} \\
\hline$f_{1}$ & 6.68 & 5.84 & 6.7 \\
\hline$f_{2}$ & 59.76 & 63.04 & 60.52 \\
\hline Similarity & YES & YES & YES \\
\hline
\end{tabular}

Although, fit factors are easy to calculate, they lack more detailed information about the actual kinetics of the release of API from tablets. At the same time, these parameters do not reflect nor account for the variability (expressed e.g. through the dispersion) associated with each dissolution profile. The $f_{2}$ is insensitive to the shape of the dissolution profiles and does not take into account the information of unequal spacing between sampling time points. In addition, fit factors are simple statistics that cannot be used to exactly (and mathematically rigorously) formulate a statistical hypothesis for assessment of dissolution similarity. Simulation results also indicated that the similarity factor is too general for making decisions about the similarity between dissolution profiles.

Aside from the analyses based on $f_{1}$ and $f_{2}$ parameters (factors), another mathematical comparison with model-independent method is performed by applying Ratio test procedures comparing the percent of dissolved test/ratio samples (PD), area under the concentration curve (AUC) as well as the average dissolution time (ADT) (Tables 3-8).

Table 3. Percent of dissolved test/ratio samples (PD) in medium $p H 4.5$.

\begin{tabular}{|c|c|c|c|c|c|c|c|c|c|}
\hline \multirow{3}{*}{$t / \min$} & \multicolumn{9}{|c|}{ PD (test/reference) } \\
\hline & \multirow{2}{*}{ T3 } & \multicolumn{2}{|c|}{$90 \%$ CI } & \multirow{2}{*}{ T 4} & \multicolumn{2}{|c|}{$90 \% \mathrm{CI}$} & \multirow{2}{*}{ T 5} & \multicolumn{2}{|c|}{$90 \% \mathrm{CI}$} \\
\hline & & low & high & & low & high & & low & high \\
\hline 10 & 1.031 & 0.934 & 1.119 & 1.137 & 1.049 & 1.224 & 1.063 & 0.976 & 1.150 \\
\hline 15 & 1.007 & 0.948 & 1.066 & 1.095 & 1.043 & 1.148 & 1.052 & 0.995 & 1.109 \\
\hline 20 & 1.037 & 0.985 & 1.089 & 1.101 & 1.054 & 1.148 & 1.064 & 1.014 & 1.113 \\
\hline 30 & 1.065 & 1.008 & 1.122 & 1.110 & 1.055 & 1.166 & 1.079 & 1.025 & 1.134 \\
\hline
\end{tabular}

Table 4. Area under the concentration curve (AUC) in medium pH 4.5.

\begin{tabular}{|c|c|c|c|c|c|}
\hline & AUC & & Ratio AUC & $90 \% \mathrm{CI}$ & \\
\hline$t / \min$ & R1 & T3 & T3/R1 & low & high \\
\hline 10 & 385.533 & 397.329 & 1.031 & 0.943 & 1.119 \\
\hline 15 & 789.390 & 808.602 & 1.024 & 0.947 & 1.102 \\
\hline 20 & 1216.208 & 1244.858 & 1.024 & 0.956 & 1.091 \\
\hline 30 & 2086.346 & 2159.329 & 1.035 & 0.981 & 1.089 \\
\hline$t / \min$ & R1 & T4 & T4/R1 & low & high \\
\hline 10 & 385.533 & 438.263 & 1.137 & 1.049 & 1.224 \\
\hline 15 & 789.390 & 888.635 & 1.126 & 1.051 & 1.201 \\
\hline 20 & 1216.208 & 1357.375 & 1.116 & 1.053 & 1.179 \\
\hline 30 & 2086.346 & 2319.433 & 1.112 & 1.063 & 1.161 \\
\hline$t / \min$ & R1 & T5 & T5/R1 & low & high \\
\hline 10 & 385.533 & 409.938 & 1.063 & 0.976 & 1.150 \\
\hline 15 & 789.390 & 836.900 & 1.060 & 0.984 & 1.136 \\
\hline 20 & 1216.208 & 1288.333 & 1.059 & 0.994 & 1.125 \\
\hline 30 & 2086.346 & 2220.671 & 1.064 & 1.013 & 1.116 \\
\hline
\end{tabular}

Table 5. Average dissolution time (ADT) in medium $\mathrm{pH} 4.5$.

\begin{tabular}{|c|c|c|c|c|c|}
\hline & ADT & & Ratio ADT & $90 \%$ CI & \\
\hline$t / \min$ & R1 & T3 & T3/R1 & low & high \\
\hline 10 & 5.000 & 5.000 & 1.000 & 1.000 & 1.000 \\
\hline 15 & 5.644 & 5.511 & 0.977 & 0.910 & 1.043 \\
\hline 20 & 5.906 & 6.105 & 1.034 & 0.959 & 1.108 \\
\hline 30 & 6.033 & 6.918 & 1.147 & 0.889 & 1.404 \\
\hline
\end{tabular}




\begin{tabular}{|c|c|c|c|c|c|}
\hline$t / \min$ & R1 & T4 & T4/R1 & low & high \\
\hline 10 & 5.000 & 5.000 & 1.000 & 1.000 & 1.000 \\
\hline 15 & 5.644 & 5.409 & 0.958 & 0.892 & 1.025 \\
\hline 20 & 5.906 & 5.727 & 0.970 & 0.902 & 1.038 \\
\hline 30 & 6.033 & 6.204 & 1.028 & 0.798 & 1.259 \\
\hline$t / \min$ & R1 & T5 & T5/R1 & low & high \\
\hline 10 & 5.000 & 5.000 & 1.000 & 1.000 & 1.000 \\
\hline 15 & 5.644 & 5.594 & 0.991 & 0.923 & 1.059 \\
\hline 20 & 5.906 & 5.985 & 1.013 & 0.941 & 1.085 \\
\hline 30 & 6.033 & 6.576 & 1.090 & 0.842 & 1.338 \\
\hline
\end{tabular}

Table 6. Percent of dissolved test/ratio samples (PD) in medium pH 6.8.

\begin{tabular}{|c|c|c|c|c|c|c|c|c|c|}
\hline \multirow{3}{*}{$t / \min$} & \multicolumn{9}{|c|}{ PD (test/reference) } \\
\hline & \multirow{2}{*}{ T3 } & \multicolumn{2}{|c|}{$90 \% \mathrm{CI}$} & \multirow{2}{*}{ T4 } & \multicolumn{2}{|c|}{$90 \% \mathrm{CI}$} & \multirow{2}{*}{ T5 } & \multicolumn{2}{|c|}{$90 \% \mathrm{CI}$} \\
\hline & & low & high & & low & high & & low & high \\
\hline 10 & 1.014 & 0.968 & 1.061 & 0.984 & 0.909 & 1.058 & 1.016 & 0.934 & 1.097 \\
\hline 15 & 1.122 & 1.064 & 1.180 & 1.088 & 1.026 & 1.150 & 1.097 & 1.036 & 1.159 \\
\hline 20 & 1.080 & 1.034 & 1.125 & 1.087 & 1.039 & 1.134 & 1.093 & 1.047 & 1.139 \\
\hline 30 & 1.049 & 1.016 & 1.083 & 1.041 & 1.009 & 1.074 & 1.059 & 1.025 & 1.092 \\
\hline
\end{tabular}

Table 7. Area under the concentration curve (AUC) in medium pH 6.8.

\begin{tabular}{|c|c|c|c|c|c|}
\hline & AUC & & Ratio AUC & $90 \% \mathrm{CI}$ & \\
\hline$t / \min$ & R1 & T3 & T3/R1 & low & high \\
\hline 10 & 376.954 & 382.388 & 1.014 & 0.968 & 1.061 \\
\hline 15 & 764.500 & 796.888 & 1.042 & 0.997 & 1.087 \\
\hline 20 & 1171.858 & 1245.138 & 1.063 & 1.019 & 1.106 \\
\hline 30 & 2031.733 & 2160.263 & 1.063 & 1.024 & 1.103 \\
\hline$t / \min$ & R1 & T4 & T4/R1 & low & high \\
\hline 10 & 376.954 & 370.821 & 0.984 & 0.909 & 1.058 \\
\hline 15 & 764.500 & 772.863 & 1.011 & 0.944 & 1.078 \\
\hline 20 & 1171.858 & 1215.825 & 1.038 & 0.980 & 1.095 \\
\hline 30 & 2031.733 & 2130.129 & 1.048 & 1.004 & 1.093 \\
\hline$t / \min$ & R1 & T5 & T5/R1 & low & high \\
\hline 10 & 376.954 & 382.825 & 1.016 & 0.934 & 1.097 \\
\hline 15 & 764.500 & 792.665 & 1.037 & 0.964 & 1.110 \\
\hline 20 & 1171.858 & 1238.677 & 1.057 & 0.996 & 1.118 \\
\hline 30 & 2031.733 & 2163.098 & 1.065 & 1.018 & 1.111 \\
\hline
\end{tabular}

Table 8. Average dissolution time (ADT) in medium pH 6.8.

\begin{tabular}{|c|c|c|c|c|c|}
\hline & ADT & & Ratio ADT & $90 \% \mathrm{CI}$ & \\
\hline$t / \min$ & R1 & T3 & T3/R1 & low & high \\
\hline 10 & 5.000 & 5.000 & 1.000 & 1.000 & 1.000 \\
\hline 15 & 5.395 & 6.080 & 1.127 & 1.058 & 1.196 \\
\hline 20 & 5.940 & 6.544 & 1.102 & 1.003 & 1.200 \\
\hline 30 & 7.100 & 6.915 & 0.974 & 0.886 & 1.062 \\
\hline$t / \min$ & R1 & T4 & T4/R1 & low & high \\
\hline 10 & 5.000 & 5.000 & 1.000 & 1.000 & 1.000 \\
\hline 15 & 5.395 & 6.051 & 1.122 & 1.067 & 1.176 \\
\hline 20 & 5.940 & 6.139 & 1.033 & 0.974 & 1.093 \\
\hline 30 & 7.100 & 6.770 & 0.954 & 0.899 & 1.008 \\
\hline$t / \min$ & R1 & T5 & T5/R1 & low & high \\
\hline 10 & 5.000 & 5.000 & 1.000 & 1.000 & 1.000 \\
\hline 15 & 5.395 & 5.938 & 1.101 & 1.032 & 1.169 \\
\hline 20 & 5.940 & 6.368 & 1.072 & 0.996 & 1.178 \\
\hline 30 & 7.100 & 6.939 & 0.977 & 0.888 & 1.067 \\
\hline
\end{tabular}

From the obtained results for $\mathrm{PD}, \mathrm{AUC}$ and $\mathrm{ADT}$ ratios in both dissolution media for all test products were within the limits that are usually considered acceptable to establish the similarity between dissolution profiles. As can be seen, the $90 \%$ confidence intervals are within the acceptable limits for the considered quantities, hence the drug release curves of the reference and the test medicinal products assess the dissolution similarity. These model-independent procedures reflect only the major or minor similarities between these two profiles, and can be considered as a good tool to judge its dissolution equivalence. However, the acceptance criteria here are also more or less doubtful. Therefore, in order to provide a more accurate, statistically justified conclusion, analysis on the basis of model-independent method based on generalized statistical distance and model-dependent method, coupled to multivariate statistical approach were accomplished.

For the purpose of the present study, the multivariate statistical analysis using both the non-transformed data for the fitting parameters, as well as the natural logarithm $(\ln )$ transformed data is performed. This was done in order to clarify the possible ambiguity in the assumption for the multivariate normal distribution of the model parameters, which has been discussed to some extent in the literature $[9,10]$. However, in the presently studied case, conclusive results both with transformed and non-transformed model parameters are obtained.

\subsection{Multivariate Model-independent Approach Based on Generalized Statistical Distance}

In a case of intra-batch (within-batch) variability, it is generally recommended that multivariate statistical methods be judged on the in vitro similarity between the dissolution profiles of the test and the reference medicinal product. Therefore, a multivariate confidence region procedure, based on $90 \%$ confidence intervals of the generalized statistical distance between the variables is carried out. Adopting a multivariate approach, the variability and correlation structure of the compiled sets of data are taken into account, which has some well-documented advantaged over the more 
conventional (and easier to apply) univariate procedures.

Upon collection or computation of the data, the phases that follow up in the course of the actual multivariate similarity test are: definition of the similarity region in terms of the maximum value of the multivariate statistical distance (MSD), expressed in the form of limiting (or maximum) value of the Mahalanobis distance; computation of the Mahalanobis distance as a measure of MSD between reference and test batches; normalization of the squared MSD $-D_{M}^{2}$ to the Hotelling $\mathrm{T}^{2}$ statistics by the scaling factor $\mathrm{K}$, to enable direct comparison to the standard F-distribution; assumption of a multivariate normal distribution of the parameters, subsequent definition of the $90 \%$ confidence region for the true difference between mean vectors; comparison of the upper limit of the confidence region to the similarity limit expressed as $\mathrm{D}_{\mathrm{M}, \mathrm{lim}}$. and derivation of a final conclusion concerning the similarity of data vectors.

The method that is actually adopted in the present study closely follows the approach derived by Tsong et al.

Comparison of dissolution profile using a multivariate method based on a statistical standardized confidence zone of the Mahalanobis distance, is performed to assess if two dissolution sets of data belong to the same initial population. This standardization means that MSD is dependent on variance and covariance estimates and can be thought of as a multivariate analogue of the two one-sided t-test procedure used in the assessment of average bioequivalence. MSD is the multi-dimensional generalization of the idea of expressing the distance between two points using standard deviation as the unit of measurement [9].

The method is a powerful statistical technique that can be implemented through analysis of variance and multivariate statistical procedures.

\subsection{Multivariate Model-dependent Approach}

As mentioned in the methodology section, on the basis on the coefficient of determination $\left(\mathrm{R}^{2}\right)$ as well as the AIC parameter, the best fit to the reference product experimental data was achieved with the Weibull model function.

In this case, out of the original solubility data in the case of the test and reference samples, we have derived the set of $\alpha$ and $\beta$ parameters for each set of measurements (each $\mathrm{F}=\mathrm{f}(t)$ curve). The mean (average) - vectors and the covariance matrices are further computed in a manner analogous to that described by (6) - (8) and the pooled covariance matrix is computed by (9) and the Mahalanobis distance by (10). The limiting value $-\mathrm{D}_{\mathrm{M}, \mathrm{lim}}$. ("the similarity limit") is in this case computed by defining a $1 \times 2$ vector $\boldsymbol{x}_{\text {lim. }}$, the elements of which are the empirically defined limits of each of the fitting parameters expressed as maxima of the tolerable average distances of the studied parameters. Again, it is assumed that the data follow multivariate normal distribution, and the $90 \%$ confidence region for the "true" difference between the population-based mean vectors $\mu_{\mathrm{T}}-\mu_{\mathrm{R}}$ is subsequently calculated from the condition (12). The final conclusion concerning the similarity of the vectors $\mathrm{x}_{\mathrm{R}}$ and $\mathrm{x}_{\mathrm{T}}$ is at the end derived by checking if the condition (15) is fulfilled, where $D_{\mathrm{M}}^{\mathrm{H}}$ has been defined by the confidence region (14). As the Weibull function is an empirical model function, not deducted directly from any fundamental kinetic model, it has been a subject of certain criticism. For example, as it does not adequately characterize the dissolution kinetic properties of the drug. Although, Weibull distribution cannot adequately characterize the dissolution kinetic properties of the drug, and perhaps the process itself, it can certainly describe the dissolution curve in terms of applicable parameters. In other words, though it might not be physically justifiable in terms of a particular kinetics model, it is mathematically convenient. At the same time, it is rather flexible, while the number of adjustable parameters is only two. On the other hand, it enables accurate determination of parameters by non-linear least-squares fitting to the experimental data, which are further subjected to multivariate statistical analyses. Table 9 and 10 summarize the results of multivariate statistical analyses of the Weibull function parameters in terms of the computed Mahalanobis distance as a generalized statistical distance. Following Tsong et al. [13], as well as the current guidelines recommendations, two dissolution curves (profiles) are regarded as similar if the upper value of $\mathrm{CI}$ is less or equal to the similarity limit expressed as the predefined (i.e. recalculated) maximum value of the Mahalanobis distance $\mathrm{D}_{\mathrm{M}, \max }$.

Table 9. Mahalanobis distance between reference $(R)$ and test $(T)$ medicinal products in medium $p H 4.5$.

\begin{tabular}{lllllll}
\hline & $\boldsymbol{K}$ & $\mathbf{F}(\mathbf{p}, \mathbf{n 1}+\mathbf{n 2}-\mathbf{p}-\mathbf{1 , 0 . 9 0})$ & $\mathbf{D}_{\mathbf{M}}$ & $\mathbf{9 0 \%}$ CI-low & $\mathbf{9 0 \%}$ CI-high & DM $_{\boldsymbol{m}_{\max }}$ \\
\hline T3 & \multirow{3}{*}{2.8636} & 2.5746 & 0.9662 & 0.0180 & 1.9144 & \\
T4 & & 0.4422 & -0.5060 & 1.3903 & 9.1330 \\
T5 & & 0.8564 & -0.0918 & 1.8046 & \\
\hline
\end{tabular}

Table 10. Mahalanobis distance between reference $(R)$ and test $(T)$ medicinal products in medium $p H$ 6.8.

\begin{tabular}{lllllll}
\hline & $\boldsymbol{K}$ & $\mathbf{F}(\mathbf{p}, \mathbf{n 1}+\mathbf{n 2}-\mathbf{p}-\mathbf{1 , 0 . 9 0})$ & $\boldsymbol{D}_{\mathbf{M}}$ & $\mathbf{9 0 \%}$ CI-low & $\mathbf{9 0 \%}$ CI-high & $\boldsymbol{D}_{\mathrm{M}, \max .}$ \\
\hline T3 & & 1.006 & 0.0525 & 1.9488 & \\
T4 & \multirow{2}{*}{2.8636} & 2.5746 & 1.0242 & 0.0761 & 1.9724 & 10.0553 \\
T5 & & 1.4956 & 0.5474 & 2.4438 & \\
\hline
\end{tabular}

\section{Conclusion}

The similarity testing of dissolution profile on the highest strength of the Bisoprolol film-coated tablets were evaluated using optimized statistical approach based on multivariate release models. The obtained results from the comparative dissolution analysis indicated on a significant similarity of the 
in vitro dissolution profiles between the Bisoprolol film-coated tablets and the reference medicinal product.

The multivariate statistic unambiguously provides extra arguments in making the decision of significant similarity between the test and the reference medicinal product.

The established combined multivariate statistical method is a fast, affordable and reliable protocol with clear acceptance criteria for examining the dissolution profile of generic medicines, reducing costs and time for submitting a registration dossier. Moreover, implemented statistical methods can be considered as a regulatory accepted concept for evaluation of in vitro similarity of generic medicines and can be regulatory relief for the registration of oral solid immediate-release formulations containing BCS classes 1 and 3 drugs. This can be in particular helpful in case of scale up or modification of strength, change which anyhow occur during the development phase.

\section{Acknowledgements}

We thank our colleague Dr. Ema Kikovska-Stojanovska for the professional support and scientific input of the manuscript.

\section{References}

[1] M. C. Gohel, M. K. Panchal, Comparison of in vitro dissolution profiles using a novel, model-independent approach, Pharm. Technol. 24 (2000) 92-102.

[2] J. W. Moore, H. H. Flanner, Mathematical comparison of dissolution profiles, Pharm. Technol. 20 (1996) 64-74.

[3] R. Cristofoletti, J. B. Dressman, Dissolution Methods to Increasing Discriminatory Power of In Vitro Dissolution Testing for Ibuprofen Free Acid and Its Salts, J. Pharm Sci. 106 (2017) 92-99.

[4] J. E. Polli, G. S. Rekhi, L. L. Augsburger, V. P. Shah, Methods to compare dissolution profiles and a rationale for wide dissolution specifications for metoprolol tartrate tablets, J. Pharm. Sci. 86 (1997) 690-700.

[5] FDA Guidance for industry: Dissolution testing of immediate release solid oral dosage forms, In. Rockville, MD (1997).

[6] FDA Guidance for industry: SUPAC-MR: Modified release solid oral dosage forms scale-up and post approval changes: chemistry, manufacturing and controls; in vitro dissolution testing and in vivo bioequivalence documentation, In. Rockville, MD (1997).

[7] European Medicines Agency, EMEA Guideline on the investigation of bioequivalence (CPMP/EWP/QWP/1401/98 Rev. 1/ Corr), London, (2010).

[8] M. E. Ruiz, M. G. Volonté, Biopharmaceutical Relevance of Dissolution Profile Comparison: Proposal of a Combined Approach, Dissolution Technologies, 21 (2014) 32-43.

[9] T. O'Hara, A. Dunne, J. Butler, J. Devane, A review of methods used to compare dissolution profile data, Pharm. Sci. Technol. To. 1 (1998) 214-223.

[10] V. Pillay, R. Fassihi, Evaluation and comparison of dissolution data derived from different modified release dosage forms: an alternative method, J. Control. Release 55 (1998) 45-55.

[11] US Pharmacopoeia 6th Edition, USP-31- NF 26, Vol 3: 3526, Monograph for Hydrochlorothiazide and Bisorprolol Tablets (2008).

[12] V. P. Shah, Y. Tsong, P. Sathe, J. P. Liu, In vitro dissolution profile comparison--statistics and analysis of the similarity factor, f2, Pharm Res. 15 (1998) 889-96.

[13] Y. Tsong, T. Hammerstrom, P. Sathe, V. P. Shah, Statistical assessment of mean differences between two dissolution data sets, Therapeutic Innovation and Regulatory Science 30 (1996) 1105-1112.

[14] N. Yuksel, A. E. Kanik, T. Baykara, Comparison of in vitro dissolution profiles by ANOVA-based, model-dependent and model-independent methods, Int. J. Pharm. 209 (2000) 57-67.

[15] H. Saranadasa, K. Krishnamoorthy, A multivariate test for similarity of two dissolution profiles, J. Biopharm. Stat. 15 (2005) 265.

[16] P. M. Sathe, Y. Tsong, V. P. Shah, In-vitro dissolution profile comparison: statistics and analysis, model dependent approach, Pharm. Res. 13 (1996) 1799-803.

[17] OriginPro 9.0.0, OriginLab Corporation (1991-2013).

[18] Mathematica 10.2.0.0, Wolfram Research, Inc. (1988-2015).

[19] Microsoft EXCEL 2007, Microsoft Corporation.

[20] V. Raju, K. V. R. Murthy, Development and Validation of New Discriminative Dissolution Method for Carvedilol Tablets, Indian J Pharm Sci. 73 (2011) 527-536. 